montseny

anarchismo

landauer

$$
\text { giustizia }
$$

femminismo

okin

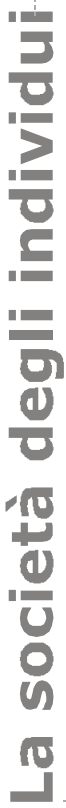

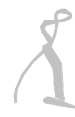

54

$2015 / 3$

FrancoAngeli 
Direttore: Ferruccio Andolfi.

Segreteria di redazione: Simona Del Bono, Donatella Gorreta, Italo Testa.

Redazione: Silvano Allasia, Simona Bertolini, Elio Canali, Thomas Casadei, Marco Deriu, Antonio Freddi, Sandra Manzi-Manzi, Chiara Marchetti, Teresa Paciariello, Corrado Piroddi, Anna Maria Ricucci, Marina Savi, Mauro Simonazzi, Timothy Tambassi, Chiara Tortora.

Comitato editoriale: Nicola Antonetti, Laura Boella, Alessandro Bosi, Paolo Costa, Enrico Donaggio, Roberto Escobar, Alessandro Ferrara, Rino Genovese, Marco Ingrosso, Sergio Manghi, Elena Pulcini, Loredana Sciolla, Alberto Siclari, Silvia Vegetti Finzi.

Comitato scientifico internazionale: Gabriel Amengual, Zygmunt Bauman, Thomas Baumeister, Ronald Beiner, Fina Birulés, Nancy Fraser, Ágnes Heller, Axel Honneth, Charles Larmore, Steven Lukes, Emmanuel Renault, Jordi Riba, Fernando Savater, Jacques Texier, Carlos Thiebaut, Adriana Veríssimo Serrao, Maurice Weyemberg.

Consulenti e collaboratori: Angela Ales-Bello, Carlo Altini, Andrea Borsari, Francesca Brezzi, Rosa Calcaterra, Antonella Cutro, Annamaria Contini, Franco Crespi, Marcella D'Abbiero, Dimitri D'Andrea, Fulvia De Luise, Giuseppe Farinetti, Francesco Fistetti, Gregor Fitzi, Marisa Forcina, Vittoria Franco, Wolfgang Huemer, Antonino Laganà, Carmen Leccardi, Anna Mandich, Aldo Meccariello, Rita Messori, Stefano Petrucciani, Gianfranco Ragona, Annamaria Rivera, Fabio Rossi, Davide Sparti, Gabriella Turnaturi, Nadia Urbinati, Maria Luisa Wandruszka, Adriano Zamperini.

Tutti gli articoli inviati alla rivista sono valutati da referee anonimi

Direzione e redazione: Dipartimento di Filosofia - Via D’Azeglio 85 - 43100 Parma - Tel. 0521902340. Fax: 902551. e-mail: ginestra@unipr.it Sito: www.laginestra.unipr.it

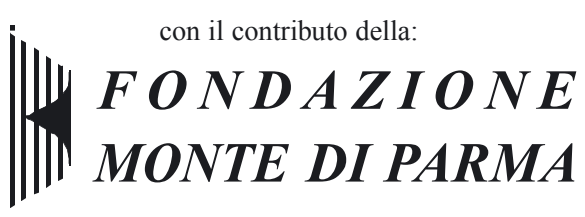

Progetto grafico: Rossana Capasso.

Amministrazione, distribuzione, abbonamenti: FrancoAngeli, V.le Monza 106 - 20127 Milano Casella postale 17175, 20100 Milano - Tel.02-2837141 - www.riviste@francoangeli.it

L'opera, comprese tutte le sue parti, è tutelata dalla legge sui diritti d'autore. Sono vietate e sanzionate (se non espressamente autorizzate) la riproduzione in ogni modo e forma (comprese le fotocopie, la scansione, la memorizzazione elettronica) e la comunicazione (ivi inclusi a titolo esemplificativo ma non esaustivo: la distribuzione, l'adattamento, la traduzione e la rielaborazione, anche a mezzo di canali digitali interattivi e con qualsiasi modalità attualmente nota od in futuro sviluppata).

Le fotocopie per uso personale del lettore possono essere effettuate nei limiti del $15 \%$ di ciascun fascicolo dietro pagamento alla SIAE del compenso previsto dall'art. 68, commi 4 e 5, della legge 22 aprile $1941 \mathrm{n}$. 633. Le fotocopie effettuate per finalità di carattere professionale, economico o commerciale o comunque per uso diverso da quello personale, possono essere effettuate a seguito di specifica autorizzazione rilasciata da CLEARedi, Centro Licenze e Autorizzazioni per le Riproduzioni Editoriali (www.clearedi.org; e-mail autorizzazioni@clearedi.org).

In caso di copia digitale, l'Utente nel momento in cui effettua il download dell'opera accetta tutte le condizioni della licenza d'uso dell'opera previste e comunicate sul sito $w w w$ francoangeli.it.

Autorizzazione del Tribunale di Parma n. 25 del 25 ottobre 1997 - Quadrimestrale - Direttore responsabile: Ferruccio Andolfi - Poste Italiane Spa - Sped. in Abb. Post. - D.L. 353/2003 (conv. in L. 27/02/2004 n. 46) art. 1, comma 1, DCB Milano

Copyright (C) 2016 by FrancoAngeli s.r.l. - Stampa: Digital Print Service srl - sede legale: via dell'Annunciata 27, 20121 Milano; sedi operative: via Torricelli 9, 20090 Segrate (MI) e via Merano 18, 20127 Milano.

III quadrimestre 2015 - Finito di stampare nel mese di febbraio 2016. 


\section{SOMMARIO}

\section{ANARCHISMI}

7 Gianfranco Ragona

Il pensiero anarchico e la politica

In luogo di un'introduzione

20 Claudio Venza

Individuo e società nell'anarchismo spagnolo

Il pensiero di Federica Montseny

33 Marco Cossutta

Lineamenti per un diritto anarchico

45 Franco Melandri e Salvo Vaccaro

Post-anarchismo in discussione

$57 \quad$ Pietro Adamo

L'anarchismo post-classico e i nuovi movimenti

\section{ArChivio}

75 Gustav Landauer

Pëtr Kropotkin

\section{FEMMINISMO E DIRITTI}

95 Ingrid Salvatore

Susan Moller Okin

Un profilo critico 


\section{RiCONOSCIMENTO}

109 Davide Sparti

"Che tipo è??

Il riconoscimento tra identificazione e descrizione

\section{FiLOSOFIA SOCIALE}

129 Leonard Mazzone

Indignazione democratica

Da pazienti ad attori morali

\section{NOTE DI LETTURA}

151 Luciano Lanza, L'anarchismo oggi. Un pensiero necessario (Timothy Tambassi); Pierre Rosanvallon, La legittimità democratica (Corrado Piroddi); Guido Mazzoni, I destini generali (Paolo Costa); Pier Cesare Bori, È una storia vera? Le tesi storiche dell'Uomo Mosè e la religione monoteistica di Sigmund Freud (Thomas Casadei)

\section{Abstract E AUTORI}

Le immagini contenute in questo numero riproducono alcuni dei ritratti esposti nel 2014 al MACRO (Museo d'Arte Contemporanea Roma) per "FotoGrafia - Festival Internazionale di Roma", nell'ambito di un progetto di ricerca curato da Eugenio Lo Sardo e Manola Ida Venzo. Si tratta di fotografie segnaletiche di anarchici del periodo 1870-1914, provenienti dall'Archivio di Stato di Roma. 


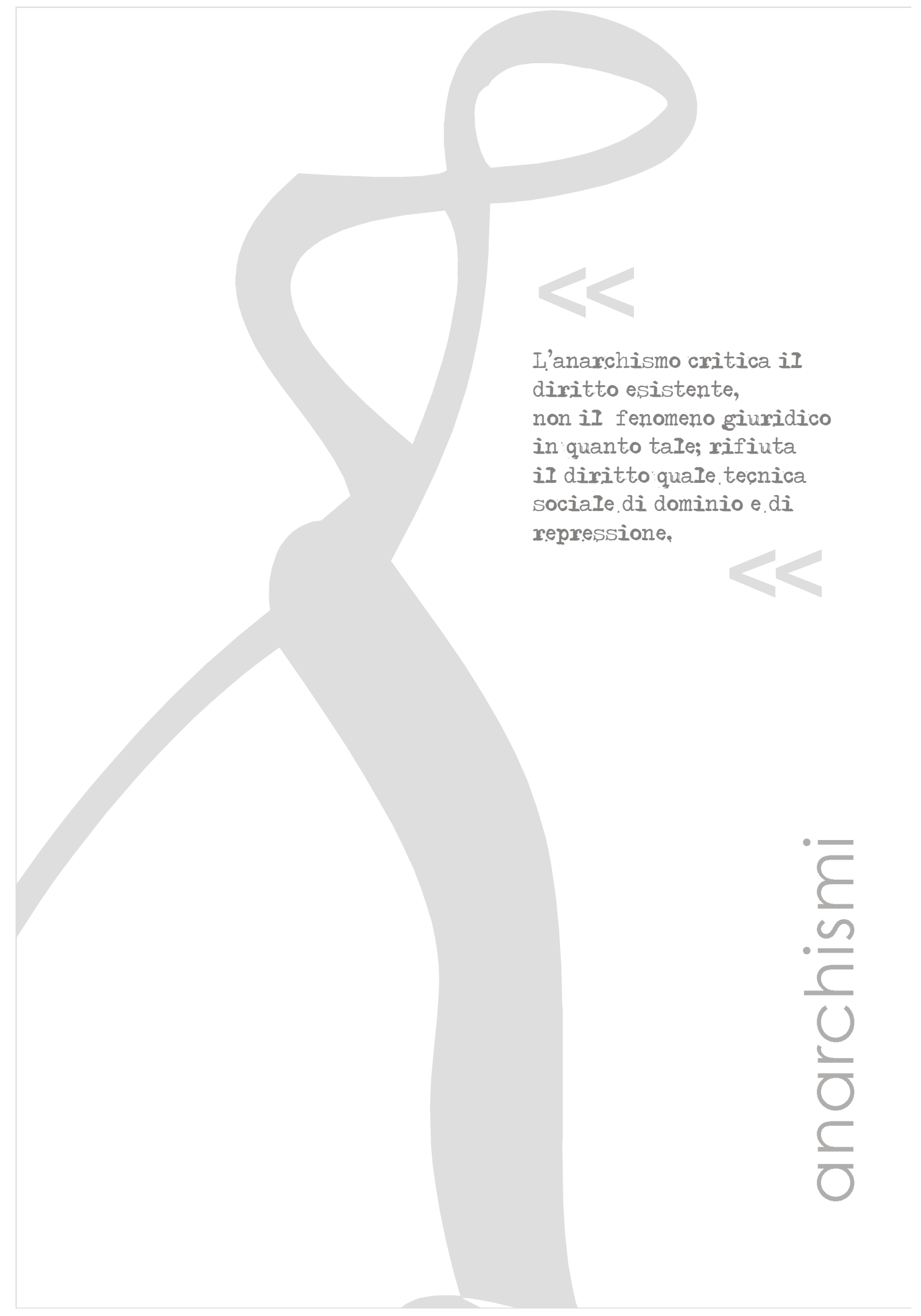




\title{
Post-anarchismo in discussione
}

\author{
Franco Melandri e Salvo Vaccaro
}

\begin{abstract}
Da un po' di anni a questa parte, negli ambienti culturali e politici più attenti ai movimenti e alle idee anarchico-libertarie dell'area anglosassone e francese, si è cominciato a discutere di "post-anarchismo», ciò̀ di un insieme di analisi e di proposte teorico-politiche che, muovendo dall'evidenziazione delle insufficienze e delle criticità via via emerse nelle teorie e nelle pratiche del movimento anarchico, cerca di ripensarne tanto la logica profonda, quanto le proposte e le modalità politiche e pratiche. A fornire il supporto concettuale e l'ossatura teorica di questo ripensamento sono soprattutto le elaborazioni che il pensiero post-strutturalista francese imperniato sulle opere di Michel Foucault, Gilles Deleuze, Jacques Derrida, Jacques Lacan - ha rivolto ai concetti, e alle pratiche ad essi correlate, di razionalità, di soggetto, di potere. Quel che il postanarchismo si propone, insomma, è un compito complesso, perché deve cercare di tenere insieme una tradizione teorico-politica ormai bicentenaria ed elaborazioni radicalmente critiche verso le categorie con cui la modernità, e l'anarchismo con essa, si è pensata e realizzata. Del tema, infatti, si è spesso occupata la rivista "Libertaria”, la cui redazione continua a seguire e sollecitare le riflessioni sulla questione anche ora che la rivista si è trasformata in un annuario, edito da Mimesis. A questo lavoro di " $\mathrm{Li}$ bertaria" hanno fatto da pendant soprattutto le edizioni Eleuthera di Milano, che hanno via via pubblicato Anarchismo e post-strutturalismo di Todd May (1998) - traduzione italiana di The political Philosophy of Poststructuralist Anarchism, uno dei testi seminali del post-anarchismo - e due importanti antologie, Pensare altrimenti (2011) e Agire altrimenti (2014), ambedue curate da Salvo Vaccaro.
\end{abstract}

Del post-anarchismo e delle questioni che esso fa emergere, Vaccarocollaboratore di varie testate anarchiche e autore o curatore anche di vari volumi su e di Nietzsche, Foucault, Deleuze, Adorno, Chomsky - discute nelle pagine seguenti con Franco Melandri, anch'egli collaboratore di lunga data di varie pubblicazioni anarchiche e libertarie e già redattore della rivista culturale "Una città". 
Franco Melandri - Per cercare di comprendere il senso e l'eventuale novità del cosiddetto post-anarchismo, credo sia innanzitutto necessario chiarire perché gli apporti della French Theory dovrebbero essere importanti per l'anarchismo in quanto tale e dove essi si distacchino in modo più significativo dalle concezioni anarchiche 'classiche'...

Salvo Vaccaro - La risposta alla tua domanda credo stia proprio nel motivo per cui ho curato Pensare altrimenti e Agire altrimenti. Questa operazione, infatti, nasce dall'intenzione di un anarchico di saggiare quanto gli apporti di pensatori quali Foucault o Deleuze - che sicuramente danno chiavi importanti per interpretare e comprendere i tempi presenti e hanno segnato il pensiero contemporaneo - possano essere integrati, 'digeriti', sotto un segno di intelligibilità anarchico. È sicuramente un'impresa eminentemente filosofica, ma risponde anche al fatto incontrovertibile che chi, come me, ha pensato di essere anarchico - o chi, al contrario, ha rifiutato l'anarchismo -, l'ha fatto a partire da un'idea forte del mondo, perché è indubbio che non sia possibile e non sia pensabile che vi sia alcuna azione sociale che non faccia perno, consapevolmente o meno, su una visione del mondo, quindi su una filosofia. Da questa necessità non può prescindere nemmeno l'anarchismo, perché è impossibile che una proposta anarchica per il XXI secolo (esattamente come una proposta anarchica per il XX o per il XIX secolo) possa essere pensata e sperimentata - in questo gioco costante di rimbalzo all'infinito tra chi pensa, chi pratica, chi riflette sulle pratiche, chi eccede il pensiero e pratica cose diverse e viceversa - senza una visione del mondo e del nostro stare nel mondo. Il nocciolo della questione è il fatto che a definire l'anarchismo è sicuramente la ricerca del massimo di libertà e insieme del massimo di uguaglianza; quindi esso si pone nella massima distanza dal liberalismo, perché in questo se si è troppo liberi non si è affatto uguali, ma si pone anche nella massima distanza dal comunismo, perché se siamo tutti uguali non siamo liberi; in questo modo, si definisce come una terza impostazione teorico-politica che certamente, come il liberalismo e il comunismo, nasce dall'Illuminismo, ma che è anche radicalmente eccedente l'Illuminismo stesso, perché si alimenta pure di pensieri di origine stoica, libertina, non-conformista, eccetera.

Il nesso fra questo percorso e quel che attraversa il nostro tempo, tuttavia, non è più percepibile nel bagaglio del movimento anarchico storico, non è emerso dai suoi presupposti concettuali, e proprio questo rende ancor più necessario farlo emergere. Dalla mia prospettiva, come ho detto, i pensieri che meglio colgono lo stare al mondo nel XXI secolo sono appunto quelli elaborati dai pensatori francesi cosiddetti post-strutturalisti. Sono essi che, oltre a tanti altri meriti specifici, hanno condensato un insieme di teo- 
resi che giudico essere il punto migliore da cui muovere proprio per cercare di elaborare oggi le proposizioni anarchiche di libertà e giustizia sociale. Questo non vuole dire ovviamente che non vi possano essere pure altre prospettive ugualmente interessanti; tuttavia ritengo evidente come, soprattutto sul piano teorico, l'anarchismo arrivato fino a noi si porti dietro una serie di sclerosi irriflesse. Sclerosi che, oltretutto, non sono state risolte nemmeno nella pratica, perché alla prova dei fatti e a parte il periodo 1936-37 in Spagna, durante la Guerra civile, l'anarchismo fino a oggi non è riuscito ad attuare una propria visibilità politica concreta. Ci sarà un motivo? Certo si può rispondere che, nelle grandi lotte sociali come in qualsiasi scontro, si può essere sconfitti, o che gli altri, in un dato contesto, sono stati più bravi, ma questo non toglie che trovi ineludibile interrogarmi, da anarchico, sulle insufficienze interne dell'anarchismo, senza colpevolizzazioni, ma anche senza trarre facili consolazioni dalla considerazione che, comunque, anche gli altri hanno sbagliato.

F. M. - Todd May, nel suo saggio contenuto in Pensare altrimenti, sottolinea positivamente che l'anarchismo è un umanesimo, cosa sicuramente vera, ma non mi sembra che colga fino in fondo il fatto che proprio questo umanesimo confligge con tutta la filosofia post-strutturalista. Questa, infatti, dimostra come tutto quel che chiamiamo 'umano' altro non sia che una costruzione socio-storica, per cui anche la razionalità e quanto da essa deriva - quindi la convinzione che i processi storici, sociali, culturali, siano in qualche modo governabili e guidabili dalla razionalità stessa - tradiscono un senso di fondo e una concezione costruttivista e quindi autoritaria, almeno potenzialmente. Inoltre, non c'è dubbio che l'intera concezione anarchica si regga su una meta-narrazione, quella che legge la storia e la società come tensione e luogo per cui si dovrebbe, o potrebbe, andare da una società segnata dal dominio alla società anarchica, che finalmente aprirebbe «le magnifiche sorti, e progressive», mentre, al contrario, Deleuze, Derrida, Lacan, destrutturano ogni meta-narrazione...

S. V. - La questione, in effetti, è centrale e, per provare a dirimerla, innanzitutto occorre distinguere fra «narrazione», «meta-narrazione»e «conarrazione». La meta-narrazione è una prospettiva che, lo dice la parola stessa, si pone come superiore a ogni singola narrazione e attribuisce a quest'ultima un attestato di autenticità o un regime di verità. Almeno da $L a$ condizione post-moderna di Jean-François Lyotard, 'manifesto' del postmoderno, la possibilità stessa di una meta-narrazione, e con essa la modernità, è però finita. Oggi non c'è più nessuno in grado di darci una narrazione 'vera', cioè uno sguardo panoramico, di fatto disincarnato e onnicomprensivo. Deleuze e Derrida, infatti, dimostrano coi loro studi che ogni 
meta-narrazione, a partire da quella della razionalità così come è emersa con e dall'Illuminismo, altro non è, al di là delle sue pretese universalistiche, che una narrazione tra le altre, sicché il solo parlare di narrazione, al singolare, è un abuso linguistico, visto che esistono esclusivamente le narrazioni, al plurale. Queste, però, non solo si danno sempre al singolare, ma anche, come verifichiamo pure empiricamente, si scambiano fra loro. Se questo è vero, ne consegue un interrogativo: queste narrazioni plurali e scambievoli possono pure esistere come co-narrazioni, cioè come narrazioni che si dispiegano intrecciandosi, senza però istituirsi e fossilizzarsi su scala verticale? Secondo me sì, perché le co-narrazioni non infrangono l'irriducibile varietà delle narrazioni, non ricadono nella trappola di ridurre la pluralità delle narrazioni in un gioco di verità, ossia in un dispositivo che le mette su una scala di priorità per cui c'è una verità che era vera ieri, una che è vera oggi e una che magari sarà vera fra un secolo.

In quest'ottica, per esempio, il fatto che l'anarchismo possa narrare se stesso ma anche modificarsi, integrando altre narrazioni, per me non è un problema, nemmeno un problema di identità, anche perché l'anarchismo dovrebbe avere una diffidenza 'genetica' nei confronti di un concetto forte di identità di sé, quindi anche verso i modi con cui esso si è storicamente dato.

Il pensiero anarchico valorizza al massimo la libertà e l'eguaglianza, ma non possiamo negare che una certa concezione della libertà e dell'eguaglianza, dai Lumi in poi, abbia dato luogo a catastrofi e ad effetti deleteri inenarrabili. Dalla Germania dei Lumi, infatti, è derivato il nazismo, dall'Illuminismo inglese è certamente derivata la democrazia americana, ma è stato proprio quel liberalismo che ha attuato il genocidio dei pellerossa o l'imperialismo coloniale. Insomma, come hanno sostenuto Adorno e Horkheimer, nell' Illuminismo e in quanto ne discende vive intrinseca una «dialettica del male», un male che ritorna, che non soltanto non è in alcun modo eliminabile, ma non è nemmeno arginabile una volta per tutte. Ora, a me sembra che, da Adorno ai teorici del post-strutturalismo, l'aver sottolineato l'aspetto del negativo, cioè la critica, senza tuttavia offrire, in positivo, una teoria del mondo rivoluzionato sia di per sé già una garanzia contro questa dialettica intrinseca. Infatti, se il positivo, sinteticamente e brutalmente detto, conduce anche al genocidio, all'annichilimento, alla shoah, non resta che rimanere ancorati al piano del negativo, un piano nel quale l'anarchismo dovrebbe collocarsi agevolmente, fin dal suo etimo: critica dell'autorità, critica di ogni principio totalizzante, il che vuol dire che non possiamo costruire nessuna meta-narrazione, nessuna filosofia normativa.

Peraltro, non bisogna correre il rischio di trasformare l'anarchismo soltanto in una filosofia, esso è anche una filosofia, ma soprattutto movimento 
di esseri umani, che agiscono seguendo le loro aspirazioni, senza necessariamente rendersi conto di quale sia l'orizzonte meta-storico di questo loro agire. Tutto questo, se certo implica che non possiamo fare una teoria dell'anarchismo data una volta per tutte, d'altra parte non significa però che non possiamo attuare delle sperimentazioni teorico-pratiche di quelle che sono le nostre pratiche valorizzate.

F. M. - Sicuramente sono possibili, e si danno empiricamente, delle conarrazioni, tuttavia, se io vivo una certa esperienza in un certo modo e tu la vivi in un altro modo, certo ce la possiamo raccontare vicendevolmente, ma ciò facendo succedono due cose: o prendiamo atto della differenza e tutto si ferma lì - quindi la differenza si dà come tale, è riconosciuta, ma permette al massimo un accordo empirico, il quale oltretutto, proprio perché del tutto evenemenziale, semplicemente accade e non permette neppure di essere detto come tale -; oppure ambedue riconosciamo che i nostri rispettivi modi di stare nel mondo possono integrarsi in una immagine, in una idea/modalità, ulteriore, che li comprende entrambi nella loro differenza e così, però, anche li supera. C'è poi da aggiungere che si devono fare i conti non con l'umanità che si vorrebbe, ma con quella che c'è e l'umanità che c'è, per fare un esempio banale, ha i Gandhi, ma ha anche i Bin Laden, e mentre i Gandhi sono disponibili a discutere con i Bin Laden, i Bin Laden non mi pare vogliano discutere con i Gandhi...

S. V. - In chiave anarchica, a tal proposito, rintracciamo una soluzione teorica e una soluzione politica. Dal punto di vista teorico, va sempre tenuto presente che l'anarchismo non è, o non dovrebbe essere, una teoria del mondo armonioso, mentre è un'opzione di organizzazione della società o, per meglio dire, un'organizzazione di legami societari in senso orizzontale, non gerarchico, non autoritario. Un'organizzazione che, oltretutto, concepisce la società non come un'unità, ma come una pluralità. Per cui, quando due o più differenze non sono conciliabili, le società si smembrano. Non occorre convivere tutti nella medesima società, perché, inversamente, è chiaro che devi trovare una formula per ridurre al silenzio, o per inglobare, la narrazione che non ti piace. Tutto questo, però, è essenzialmente un problema politico. Se, infatti, accetto l'idea che non vi possano essere che molte narrazioni, è solo dal punto di vista politico che si pone il problema di neutralizzare, magari cercando un modo diverso dal rapporto di forza brutale, chi ti vuole sterminare.

F. M. - In questo senso, certamente, la questione è essenzialmente politica ed è appunto in questo ambito che si muove la democrazia liberale. Con l'idea e la pratica della democrazia, questa almeno è l'auto-rappresen- 
tazione liberal-democratica, hai infatti la possibilità di far convivere e confrontare anche narrazioni del mondo fra loro radicalmente conflittuali...

S. V. - È vero, la democrazia lascia vivere le differenze, però soltanto entro certi limiti e soprattutto organizzandole in senso autoritario attraverso il principio di maggioranza e attraverso la forma-Stato. Il problema, in realtà, è che, come appunto dimostrano anche i filosofi francesi, la democrazia liberale non ha risolto il problema teorico di definire i lineamenti di una società che non sia unitaria. Certamente la democrazia liberale accetta che la sua Unità sia fatta di parti, ma appunto in quanto parti dell'Unità, quindi parti che abbisognano di questa, non parti che potrebbero anche fare a meno di altro al di là di esse. La democrazia, cioè, continua ad avere al suo fondo un'idea monolitica della società e appunto dà a questa idea la forma della statualità, all'interno della quale il sistema di soluzione dei conflitti è il principio della maggioranza, cioè un sistema gerarchico e verticale, anche se è contingente, perché le maggioranze possono cambiare. In quanto anarchici, al contrario, dobbiamo trovare una soluzione, tanto teorica quanto politica, che non sia quella democratico-maggioritaria. Dal punto di vista teorico, occorre smontare l'idea di società in quanto Unità, perché è solo in questo modo che possono coesistere le differenze che non riescono a conciliarsi. Ecco perché a me non piace parlare di 'società', mentre preferisco parlare di legami che si allacciano e si slacciano secondo le differenti esperienze di vita che ciascuno fa tranquillamente nella quotidianità.

F. M. - Concordo con te che quella che chiamiamo società sia perlomeno una società di società, una questione divenuta ancora più chiara da quando, a partire almeno dalla prima metà del ' 900 , sono emerse le società di massa, nelle quali, non a caso, per poter costruire un'unità si è finiti, e non si poteva non finire, nei totalitarismi. In questa condizione epocale, la questione della politica, come hai appena detto, si rivela sempre più centrale, ma proprio rispetto ad essa le teorizzazioni dei pensatori francesi hanno svolto un lavoro di tipo eminentemente distruttivo. Foucault e Derrida, infatti, hanno giustamente mostrato che la politica, almeno com'è stata finora, vive di logiche tecnico-autoritarie e, conseguentemente, di pratiche irreggimentanti e repressive, ma non hanno delineato una possibile politica 'altra'. Questo vuoto, però, non rischia di ridare forza e valore o ad un'idea machiavelliana del concetto di politica, cioè alla politica intesa solo come luogo e modo di conquista e gestione del potere, che avrebbe valore e senso in sé e per sé, oppure all'idea rousseauiano-marxista che la dimensione politica sia solo una dimensione del tutto accessoria, di cui, in fondo, si potrebbe in qualche modo fare a meno? 
S. V. - Innanzitutto chiariamo i termini della questione. Da una parte, c'è una critica ormai consolidata, cioè la critica alla politica come sfera che si è separata dai legami sociali. È la critica alla politica istituzionale fatta da Cornelius Castoriadis, il quale ha ben mostrato come la politica istituita nella forma-Stato prevarichi la stessa possibilità di stringere patti che ciascuno di noi in potenza ha in quanto differenza, poiché ognuno è unico al mondo. Dall'altra parte, oltre alla critica alla politica istituzionalizzata, è essenziale criticare anche la 'grammatica' della politica, e su questo i francesi danno contributi formidabili. Smontare la grammatica della politica, infatti, vuole dire smontare la politica come grammatica, quindi smontare quella matrice che ci dà le indicazioni su come comportarci innanzitutto come corpi, più e prima ancora che come istanze valoriali. È cioè quella grammatica che ci dice, in quanto logica e pratica consolidate, quello che possiamo fare, come lo possiamo fare, con chi e quando, ponendoci altresì un plusvalore di senso, ossia una ragione sufficiente. È questa la grammatica politica che, nell'era moderna, da Hobbes in qua, si è sforzata di giustificare chi pone queste regole e la loro vigenza, una logica che si è mantenuta anche col sorgere delle democrazie liberali. La democrazia liberale sostiene infatti che le regole del gioco sono neutrali, per cui non solo tutti potremmo giocare alla pari, ma non vi sarebbe nessun disegno preordinato e la società sarebbe «aperta», per dirla con Karl Popper. In tutte le società e culture, infatti, certamente esistono delle regole del gioco, ma solo nelle civiltà come la nostra il momento istituente di tali regole si è sempre posto come staccato e superiore alla società stessa, il che, nelle società liberali e nella nostra grammatica politica, coincide, come già si è detto, col momento rappresentativo. Ugualmente rappresentativo e separato è il potere politico, cioè l'istanza che pone il chi, il come, il quando e il perché quelle regole del gioco vadano applicate. In questo senso, smontare la grammatica della politica e la politica come grammatica significa dimostrare come il potere non abbia legittimità, quindi significa andare contro Hobbes, perché da Hobbes in poi tutte le teorie politiche hanno mirato a giustificare l'inamovibilità del potere. Foucault, Derrida, e prima anche Étienne de la Boétie, hanno cercato di dimostrare come il potere sia un dispositivo che funziona a prescindere dalle istanze di legittimazione e giustificazione. Il potere, anzi, non ha necessità di nessuna giustificazione, se non come un orpello per illudere, per attirare, per integrare, per tenere buoni, cioè come tassello dell'ordine. Decostruire la grammatica, e quindi la semantica, della politica vuole inoltre dire riconsegnare a se stesse, al loro allacciarsi e slacciarsi in modo continuo e magmatico, le relazioni sociali, le quali, essendo appunto riconsegnate a se stesse, non devono perciò essere rese trasparenti per un'istanza ulteriore. In questa riconsegna a se stesse, quindi, c'è anche la ricon- 
segna alla loro stessa consapevolezza, quindi all'auto-organizzazione, o autogoverno, dei singoli, che appunto allacciano e slacciano quelle relazioni che poi intessono la trama della società. È tutto questo che definisco politica, perché questo continuo allacciarsi e slacciarsi è un fatto profondamente, anche se non esclusivamente, pubblico, e lo è perché si manifesta in uno spazio comune, coinvolgente secondo le trame che vengono attivate o disattivate in base alle esigenze più o meno temporanee di quelle stesse singolarità. In ciò riscontro una proposta in sintonia con l'impostazione dell'anarchismo, al di là del fatto che quegli autori volessero o meno l'anarchia.

F. M. - Ma anche questa idea di tornare alle relazioni basilari fra le persone, al di là della politica e della sua grammatica, secondo me pone una serie di problemi. Il primo, più generale, è che al fondo mi pare ci sia la convinzione che esista un 'umano' al di là delle forme, delle grammatiche, quindi delle culture e degli universi simbolico-culturali, cosa che, invece, proprio le analisi critiche della French Theory dimostrano non essere possibile, perché il mostrare come ogni rapporto umano sia storicamente avvenuto attraverso delle grammatiche - che a loro volta sono storiche, mutevoli e contingenti - dice anche, allo stesso modo, che non sono possibili o pensabili rapporti umani se non, appunto, attraverso delle grammatiche. D'altra parte è proprio in virtù della grammatica che esiste un soggetto nei due sensi del termine, cioè in quello attivo di 'soggetto di' e in quello passivo di 'soggetto a' (il quale, anzi, proprio perché, è 'soggetto a', si rivela pure 'in condizione di' o 'soggetto di'). Certo rimane una grammatica e, in questo senso, un 'potere', cioè un aspetto performativo, ma è un potere che, come del resto sottolineava lo stesso Foucault, va verso l'auctoritas nel senso della sua radice lessicale, cioè di augere, far crescere...

S. V. - Certamente perché si possa narrare è necessario un linguaggio e quindi una grammatica, ma l'aspetto di cui parli tu, e verso cui guardavano i filosofi francesi di cui stiamo parlando, non è tanto performativo nel senso di autoritario, ma è, o potrebbe essere, la forma libertaria della necessità delle decisioni collettive. D'altra parte, fin da Bakunin, il concetto anarchico di libertà si accompagna a quello di responsabilità. Gli anarchici hanno sempre criticato l'idea liberale della libertà come laissez faire, perché per loro vale appunto la definizione di Bakunin: la mia libertà si completa nella libertà dell'altro e questa completezza, per non essere invasiva, deve giocarsi tutta su un piano di responsabilità, cioè nella capacità di rispondere alla libertà altrui, cosicché le libertà singole rimbalzano, per così dire, l'una con l'altra e l'una nell'altra. È chiaro che questo concetto di libertà come responsabilità implica un'auto-educazione dell'umanità, che è appunto uno degli obiettivi del pensare e della pratica anarchica, nel senso di una sottra- 
zione dall'umano così come esso è, perché subisce l'istruzione di concetti, e soprattutto di pratiche quotidiane, in cui la libertà non è per nulla concepita come responsabilità etica.

Un altro elemento da tenere presente è che questo rimbalzare delle libertà l'una nell'altra, questo completarsi vicendevolmente, non può mai pretendere di essere fondativo, come invece pretende di essere un qualsiasi potere. Il gioco delle libertà, infatti, non può essere fondativo perché nessuno di noi fonda mai nulla, e ciò per la banale ragione che tutti noi arriviamo sempre a metà di qualcosa che è iniziato già da sempre. Occorre liberarsi del problema di dover fondare il principio, dobbiamo fare a meno dell'arché, per dirla col linguaggio filosofico, e dobbiamo sbarazzarcene proprio perché, comunque, non possiamo che essere sempre nel mezzo, quindi non possiamo che 'derivare da'. D'altra parte, proprio Deleuze, riferendosi a Foucault, sottolineava che derivare non significa dipendere e questo non è un gioco di parole, un escamotage, perché 'derivare da' vuole appunto dire, come accennavi tu, essere posti in una certa condizione di possibilità.

In fondo essere liberi vuole dire aver cura di sé, per evocare ancora il fantasma di Foucault, ed in questo senso penso che con un cinese, o un arabo o un indiano, ci si possa benissimo capire. Se non curo me stesso, la responsabilità non sarà mai un habitus, una pratica della libertà, al massimo la concepirò come valore, come un dover essere che si cala sulle cose, sulla vita, dall'esterno. In questo senso, per esempio, quando un filosofo certo lontano dall'anarchismo come Emmanuel Lévinas dice che l'etica è la filosofia prima, cioè che l'etica è fondante anche della stessa possibilità dell'ontologia, dice una cosa che riguarda direttamente l'anarchismo, perché pone esattamente la questione del plurale, della pluralità empirica della vita e dei suoi modi di darsi. È proprio questa pluralità che mostra pure come non esista un altro radicalmente separato, scisso, dagli altri, ossia che siamo sempre chiamati a un terreno comune, che in qualche modo determina anche chi lo vorrebbe rifiutare.

F. M. - Proprio questo, però, implica anche che non ci si possa tirare fuori dalle dinamiche politiche quotidiane, pena l'esserne travolti. E queste dinamiche sono fatte di tanti tipi di rapporto, non ultimi i rapporti di forza e/o quelli determinati dalle istituzioni...

S. V. - Meno degli altri gli anarchici devono praticare l'assenza dal mondo. Sicuramente possiamo teorizzare che l'anarchico si assenti dallo spettacolo della politica, possiamo dire che non deve entrare nei Consigli comunali o nel Parlamento, ma non possiamo teorizzare, e tantomeno praticare, l'anarchismo come assenza dal mondo. Gli anarchici non possono neanche praticare l'indifferenza tra le varie opzioni che hanno di fronte, perché è chiaro 
che, come dicevano Malatesta o Berneri, è meglio vivere dove ci sono più ambiti di libertà. Questo non è un riconoscimento tale da porre uno scandalo teorico, perché è una semplice considerazione di buon senso riconoscere che io stia meglio dove c'è più libertà che non vivere dove c'è il gulag.

F. M. - Tu dici che la politica è una cosa e la rappresentazione della politica, la scena, è un'altra: ma la politica, in quanto conversazione, discussione e decisione pubblica su quanto riguarda tutti, in ogni caso non può fare a meno di spazi neutri...

S. V. - Innanzitutto chiariamo che «neutro» non è sinonimo di «comune», perché dire neutro rimanda all'idea che quello spazio sia terzo, cioè che esista indipendentemente da me, da te. Parlare invece di spazio comune implica che quello spazio non esista se non nel momento in cui ci sono dei conversanti che lo fanno esistere. Se così è, però, perché dobbiamo istituzionalizzare il nostro spazio comune di conversazione? Stiamo conversando? Abbiamo uno spazio istituito. Quando finiremo, questo spazio sparirà, punto e basta. I livelli di istituzionalizzazione devono dipendere dagli attori e dalle dinamiche in campo: quando queste dinamiche e questi attori esistono, esiste lo spazio comune e può durare un'ora, un mese, un anno, o indefinitamente, quando non ci sono, sparisce.

La situazione attuale, inoltre, è quella per cui, come intitola un suo libro Jean-Luc Nancy, l'essere è singolare e plurale. Questi due aggettivi vogliono denotare che a mettersi in comunicazione, a mettersi insieme, non soltanto sono sempre delle differenze, ma che esse non debbono risolversi in, o definirsi a partire da, un modello di identità umana, mentre non possono che essere differenze che si stagliano come tali e come tali vogliono rimanere, quindi non possono che essere plurali, quindi devono fluidificare e non rimanere bloccate in se stesse.

F. M. - Questa circolazione delle differenze, tuttavia, dovrà in qualche modo trovare un punto e un modo in cui, appunto, le differenze si intersechino sapendosi come tali e volendosi mantenere in quanto tali. In quest'ottica, però, uno dei rapporti più facilmente possibile, e purtroppo più frequente, è quello in cui la differenza radicale viene vista come hostis, con quel che ne consegue, cioè l'idea di Carl Schmitt che il rapporto fondamentale fra esseri umani sia quello amico/nemico...

S. V. - Credo che questa visione schmittiana debba essere cassata una volta per tutte, anche perché alle tesi di Schmitt si possono contrapporre almeno due obiezioni. Una è quella che, come è stato varie volte rilevato, in fin dei conti la diade amico/nemico non è una vera diade e non lo è perché proprio nel pensiero di Schmitt il nemico è prioritario, essendo il nemico a 
determinare colui che può essere mio amico, che quindi è tale perché è nemico del mio nemico. Quindi, a differenza del bene/male e delle altre diadi che danno il segno emblematico dell'ambito morale, qui siamo in presenza di una falsa diade, perché l'amico non è dato di per sé, è sempre derivato, 'dipende da'.

La seconda considerazione muove dall'interpretazione che Derrida fa della categoria dell'ospitalità presso i Greci.

In questo senso, la prima questione da sottolineare è che la contrapposizione non è fra l'hostis, in quanto estraneo e quindi potenzialmente nemico, e l'hospes, in quanto straniero e quindi possibile ospite, ma fra l'hostis e l'hospes, che non a caso per i Greci coincidevano, e il loro comune contrario, cioè l'inimicus, il nemico. È una questione che si vede bene, dice Derrida, già nell'Odissea, quando Ulisse, andato nell'antro del ciclope Polifemo viene da questi catturato, ma riesce ad accecarlo e a sfuggirgli dicendogli, va sottolineato, «È Nessuno che ti ha accecato e ti sconfigge».

Ma perché gli dei fanno vincere Ulisse e fanno impazzire il ciclope? Perché chi scrive l'opera è impregnato dalla cultura dell'ospitalità ed è in quest'ottica che il ciclope, cioè il barbaro che ha un unico occhio ed è quindi un panottico in miniatura, si dimostra inospitale appunto nei confronti dell'hostis che, proprio in quanto tale, non ha nome, è tutti e nessuno.

Già queste due considerazioni mostrano come la fondatività concettuale della diade hostis/hospes sia tutt'altro che dimostrata e sia anzi decostruita, la qual cosa, a sua volta, mostra come il discorso del nemico in quanto elemento fondativo della politica sia una trappola della politica stessa, non certo il suo segno distintivo. Il segno distintivo della politica, al contrario, è il saper e voler coniugare rischio e responsabilità. Questa è la politica ed è proprio per questo che essa pone una sfida angosciante, esistenziale, tale appunto in quanto essa è uno dei luoghi principali dell'ospitalità. L'ospitalità, nel senso profondo dei Greci, non può che essere incondizionata, cioè scevra dalla diffidenza, la quale, anzi, è l'effetto reiterato della smentita dell'ospitalità incondizionata, cioè del rischio che io corro quando mi apro, quando mi espongo all'altro, perché è comunque indubbio che l'altro sia sempre un rischio. Per questo, quando parliamo di libertà, dovremmo pure aggiungere che libertà, oltreché responsabilità, è anche rischio, perché nella libertà ci giochiamo la nostra esistenza.

Per questo la libertà è una sfida che è infinita, che non può essere risolta per sempre in una qualche forma di società - si chiami questa società democrazia o si chiami anche anarchia -, perché qualsiasi 'stato' di cose significa bloccare il divenire, ingessarlo, mentre l'anarchia è sempre in divenire, non sarà mai uno stato della società, ma saranno legami sociali che si allacciano e si dissolvono. 
I pensatori della French Theory, smontando le giustificazioni del potere, mostrano altresì gli inghippi che si celano in una concezione dell'anti-potere che illuministicamente si muova, come è quella dell'anarchismo tradizionale, in un'ottica di fatto sostitutiva. Quel che i filosofi post-strutturalisti mostrano in modo chiarissimo all'anarchismo, insomma, è che suo compito non è costruire una società anarchica, nel senso di una società che sostituisca al potere un anti-potere uguale e contrario, ma di lavorare per creare una società libertaria. E dico libertaria anche per mettere in luce un ulteriore problema: ci dobbiamo sempre mettere un aggettivo, perché siamo sempre intrappolati nella forca linguistica. Parafrasando Nietzsche: «Dio è morto, ma resta sempre la grammatica». Noi, allora, vogliamo una società libertaria, dando a questo termine il senso di divenire, di magma, di non istituzionalizzazione degli spazi che si aprono, spazi che non sono sfere ma contingenze, che sono particolarità e non universalità. 